\title{
Preparation, electrical properties and thermal stability of conductive polyaniline:nylon-6,6 composite films
}

\author{
M. Khalid, F. Mohammad* \\ Aligarh Muslim University, Department of Applied Chemistry, Aligarh-202002, India
}

Received 26 July 2007; accepted in revised form 29 August 2007

\begin{abstract}
The polyaniline:nylon-6,6 (PANI:Ny-6,6) composite films were prepared by diffusion process and the oxidative polymerization of aniline within the nylon-6,6 matrix. The composite films were characterized by fourier transform infrared spectroscopy (FTIR), scanning electron microscopy (SEM) as well as for their electrical properties. The surface electrical conductivity of the $\mathrm{HCl}(1 \mathrm{M})$ doped composite films increases with increase in the polyaniline content of the films. The study of electrical properties under isothermal conditions in the temperature range of $50-130^{\circ} \mathrm{C}$ showed that the composite films were stable under ambient conditions below $90^{\circ} \mathrm{C}$ in terms of DC electrical conductivity retention.
\end{abstract}

Keywords: polymer composites, electrical properties, thermal stability

\section{Introduction}

There is an explosive increase in the demand for composite materials in the last two decades, which are now available as materials with unique combination of properties. The electronic and optical properties of conducting polymer and insulating polymer matrix combined with attractive mechanical properties and processing advantages of the polymers has now attained a level of maturity consistent with a new set of opportunities to develop a wide range of application based conducting polymer composites. Thus, the composites based on conducting polymers and insulating polymers have been studied as materials for industrial products, such as rechargeable batteries, conductive coatings, light emitting diodes, gas sensors and antistatic materials [1].

Polyaniline has been widely investigated due to its low cost, easy synthesis and high as well as tailorable electrical conductivity depending upon the level of doping, environmental stability and interesting redox properties [2-5]. In spite of its several desirable properties, the insolubility in conventional solvents for processing [6] and poor mechanical strength limit its application [7].

One of the ways to overcome these demerits is to prepare conducting composites of polyaniline by chemical or electrochemical polymerization within an insulating polymer matrix $[8,9]$. It is easy to control the chemical polymerization of aniline within the insulating polymer matrix to prepare conducting composites of required electrical properties while retaining the mechanical strength of insulating polymer matrix [10-12]. In this paper, we have reported a simple chemical route to prepare polyaniline:nylon-6,6 composite films. Nylon6,6 is one of the most popular materials used as fibers and thermoplastics.

\section{Experimental}

\subsection{Reagents and chemicals}

The main chemicals used, for the preparation of composite films were: aniline, 99\% (CDH, India), 
Table 1. Preparation details of polyaniline:nylon-6,6 composites

\begin{tabular}{|l|c|c|c|c|c|c|}
\hline \multicolumn{1}{|c|}{ Sample ID } & $\begin{array}{c}\text { Temp } \\
{\left[{ }^{\circ} \mathbf{C}\right]}\end{array}$ & $\begin{array}{c}\text { Time } \\
{[\mathbf{h}]}\end{array}$ & $\begin{array}{c}\text { Weight of } \\
\text { nylon-6,6 films }[\mathbf{g}]\end{array}$ & $\begin{array}{c}\text { Amount of } \\
\text { aniline soaked [g] }\end{array}$ & $\begin{array}{c}\text { Weight of film } \\
\text { after soaking [g] }\end{array}$ & $\begin{array}{c}\text { Weight of films after } \\
\text { polymerization [g] }\end{array}$ \\
\hline PANI:Ny-6,6-(1) & 80 & 10 & 0.2658 & 0.0225 & 0.2883 & 0.2984 \\
\hline PANI:Ny-6,6-(2) & 80 & 15 & 0.2658 & 0.0450 & 0.3108 & 0.3217 \\
\hline PANI:Ny-6,6-(3) & 80 & 20 & 0.2658 & 0.0675 & 0.3323 & 0.3450 \\
\hline PANI:Ny-6,6-(4) & 80 & 25 & 0.2658 & 0.0910 & 0.3558 & 0.3682 \\
\hline PANI:Ny-6,6-(5) & 80 & 30 & 0.2658 & 0.1125 & 0.3783 & 0.3916 \\
\hline PANI:Ny-6,6-(5a) & 80 & 30 & 0.1627 & 0.0138 & 0.1765 & 0.2396 \\
\hline PANI:Ny-6,6-(5b) & 80 & 30 & 0.1694 & 0.0148 & 0.1842 & 0.2416 \\
\hline PANI:Ny-6,6-(5c) & 80 & 30 & 0.1794 & 0.0156 & 0.1960 & 0.2643 \\
\hline
\end{tabular}

nylon-6,6 (from Research, Design and Standard Organization), hydrochloric acid, 35\% (E. Merck, India), potassium persulphate, $98 \%$ (CDH, India).

\subsection{Preparation of polyaniline:nylon-6,6 composite films}

Square shaped pieces of $0.265 \mathrm{~g}$ each were cut from nylon-6,6 sheet and then pressed into thin films by applying pressure of 10 tons in an Electrically Operated Automatic Pressure Machine maintained at a temperature of $220^{\circ} \mathrm{C}$. The thickness of films varied in the range of 0.40 to $0.50 \mathrm{~mm}$. Each film was kept for soaking in $30 \mathrm{ml}$ of doubly distilled aniline for different periods of time in an air oven at $80^{\circ} \mathrm{C}$ to allow aniline diffuse into nylon-6,6 matrix. Aniline soaked films were treated with $0.1 \mathrm{M}$ potassium persulphate in $1 \mathrm{M} \mathrm{HCl}$ solution and left for 24 hours at ice temperature in order to polymerize aniline within the nylon- 6,6 matrix. Thus prepared polyaniline:nylon- 6,6 composite films were washed with doubly distilled water up to acid neutralization and undoped by treatment with excess of aqueous ammonia $(1 \mathrm{M})$ followed by washing with distilled water until the filtrate became neutral. The composite films were dried for 24 hours at $40^{\circ} \mathrm{C}$ in a dry box and then stored in desiccators for experiments. Polyaniline:nylon-6,6 composite films were doped by treating with $1 \mathrm{M} \mathrm{HCl}$ solution at room temperature for 24 hours. The preparation details are given in Table 1.

\subsection{Testing methods}

For the study of the difference in surface morphology between the parent materials and their composites, SEM micrographs were taken by LEO-435 VP SEM instrument. FTIR spectra of polyaniline: nylon-6,6 composites were recorded by NicoletteProtégé 460. DC electrical conductivity of the doped composites films was measured with increasing temperature by using a four-in-line probe technique. DC electrical conductivity $(\sigma)$ was calculated using Equations (1) and (2):

$$
\begin{aligned}
& \rho=\frac{\rho_{0}}{G_{7}(W / S)} \\
& \sigma=\left(\frac{1}{\rho}\right)
\end{aligned}
$$

where $\sigma, \rho, \rho_{0}, W$ and $S$ electrical conductivity $[\mathrm{S} / \mathrm{cm}]$, corrected resistivity $[\Omega \cdot \mathrm{cm}]$, uncorrected resistivity $[\Omega \cdot \mathrm{cm}]$, thickness of the film $[\mathrm{cm}]$ and probe spacing [cm] respectively [13]. $G_{7}(W / S)$ is a correction divisor, which is a function of thickness of the sample as well as probe spacing. The sample to be tested is placed on the base plate of four-probe arrangement and the probes were allowed to rest in the middle of the sample. A very gentle pressure is applied on the probes and then it was tightened in this position so as to avoid piercing of the probes into the samples. The arrangement was placed in electrically controlled oven. The current was passed through the outer probes and the floating potential across the inner pair of probes was measured. The power supply of the oven was then switched on, the temperature was allowed to increase gradually. The current and voltage were recorded simultaneously with a rise in the temperature.

The thermal stability of composite samples in terms of DC electrical conductivity retention was studied under isothermal conditions by using four-in-line DC electrical conductivity measuring instrument. This study was carried out at 50, 70, 90, 110 and $130^{\circ} \mathrm{C}$ on the selected composite films. The electrical conductivity measurements were done at an interval of $10 \mathrm{~min}$. 


\section{Results and discussion}

\subsection{Preparation of conducting polymer composite films}

The preparation of polyaniline:nylon-6,6 composite films was done by diffusing of aniline into nylon-6,6 matrix. During this process, some amount of nylon-6,6 was dissolved in aniline producing jelly like precipitate above $80^{\circ} \mathrm{C}$ as well as if kept in aniline for more than 30 hours at $80^{\circ} \mathrm{C}$. Therefore, the temperature and duration of soaking of aniline within the nylon-6,6 matrix were selected at 30 hours and $80^{\circ} \mathrm{C}$ respectively. The composition of composite films for the determination of variation in electrical conductivity of the composite films, due to the change in amount of nylon- 6,6 , four different samples were prepared as same temperature and time. Hence, a number of $\mathrm{HCl}$ doped polyaniline:nylon-6,6 composite film samples were prepared as detailed in Table 2.

When aniline is oxidized with $0.1 \mathrm{M}$ potassium persulphate $\left(\mathrm{K}_{2} \mathrm{~S}_{2} \mathrm{O}_{8}\right)$ in acidic media $(\mathrm{HCl})$, the protonated conducting form of polyaniline (emeraldine salt) is produced with black color [14].

Schöllhörn and Zagefka [15] have suggested a redox reaction for ammonia or amine intercalation into layered metal chalcogenides, which has been further supported by the work of Foot and Shaker [16]. On the basis of disproportionation reaction of ammonia as suggested by Mohammad [17] for the undoping of polythiophene (PTH) by water. The overall chemical reactions are given in Equations (3)-(7):

$$
\begin{aligned}
& 8 \mathrm{NH}_{3} \rightarrow 6 \mathrm{NH}_{4}^{+}+6 \mathrm{e}^{-}+\mathrm{N}_{2} \\
& \mathrm{PTH}^{+}-\mathrm{BF}_{4}^{-}+\mathrm{NH}_{4}^{+}+\mathrm{e}^{-} \longrightarrow \mathrm{PTH}+\mathrm{NH}_{4} \mathrm{BF}_{4} \\
& 6 \mathrm{H}_{2} \mathrm{O} \longrightarrow 4 \mathrm{H}_{3} \mathrm{O}^{+}+4 \mathrm{e}^{-}+\mathrm{O}_{2}
\end{aligned}
$$

$$
\mathrm{PTH}^{+}-\mathrm{BF}_{4}^{-}+\mathrm{H}_{3} \mathrm{O}^{+}+\mathrm{e}^{-} \longrightarrow \mathrm{PTH}+\mathrm{HBF}_{4}+\mathrm{H}_{2} \mathrm{O}
$$

$\mathrm{HBF}_{4} \longrightarrow \mathrm{HF}+\mathrm{BF}_{3}$

The charge neutralization reaction depends on the rate of chemical reaction between the doped polymer and undoping agent, which depends upon the reactivity of the polymer chain and basic strength of undoping agent [17]. The basic strength of water is very low, hence, it does not act as an effective undoping agent in the case of polyaniline, however, an analogous neutralization reaction for the undoping of the polyaniline component of the composites by ammonia solution may be suggested as shown by Equations (8) and (9):

$\mathrm{NH}_{3}+\mathrm{H}_{2} \mathrm{O} \rightarrow \mathrm{NH}_{4} \mathrm{OH}$

$$
\begin{aligned}
& {\left[\left(\mathrm{PANI}-n \mathrm{H}^{+}(\mathrm{Ny}-6,6)\right]\left[\left(n \mathrm{Cl}^{-}\right)\right]+n \mathrm{NH}_{4} \mathrm{OH}\right.} \\
& \rightarrow \text { PANI }(\mathrm{Ny}-6,6)+n \mathrm{NH}_{4} \mathrm{Cl}+n \mathrm{H}_{2} \mathrm{O}
\end{aligned}
$$

Recently, it has been reported that PANI:Ny-6 composite film consisted of layers, the outer layers were conducting composite layers and the inner layer was pristine nylon-6 [18]. In a similar way, it seems that polyaniline is mainly polymerized in the outer layer of the composite film (PANI:Ny-6,6) and this layers retards the diffusion of the oxidant solution to the interior side and prohibits polyaniline from being polymerized inside the film or aniline does not diffuse deep inside the polymer matrix as observed in the cross-sectional view of the films.

\begin{tabular}{|c|c|c|c|c|}
\hline \multirow{2}{*}{ Sample ID } & \multirow{2}{*}{$\begin{array}{c}\text { Amount of } \\
\text { aniline soaked [\%] }\end{array}$} & \multicolumn{2}{|c|}{ Color } & \multirow{2}{*}{$\sigma[\mathrm{S} / \mathrm{cm}]$} \\
\hline & & As-prepared & HCl doped & \\
\hline PANI:Ny-6,6-(1) & 08.4 & light green & light brown & very low \\
\hline PANI:Ny-6,6-(2) & 16.9 & light green & light brown & very low \\
\hline PANI:Ny-6,6-(3) & 25.3 & green & dark brown & $\sim 10^{-5}$ \\
\hline PANI:Ny-6,6-(4) & 33.8 & green & black & $\sim 10^{-3}$ \\
\hline PANI:Ny-6,6-(5) & 42.3 & dark green & black & $\sim 10^{-1}$ \\
\hline PANI:Ny-6,6-(5a) & 42.3 & dark green & black & $0.121 \cdot 10^{-1}$ \\
\hline PANI:Ny-6,6-(5b) & 42.3 & dark green & black & $0.262 \cdot 10^{-1}$ \\
\hline PANI:Ny-6,6-(5c) & 42.3 & dark green & black & $0.134 \cdot 10^{-1}$ \\
\hline
\end{tabular}

\subsection{Characterization}

\subsubsection{SEM studies}

Figure 1 shows the SEM photographs of polyaniline, nylon-6,6 and polyaniline:nylon- 6,6 compos-

Table 2. Preparation details of $\mathrm{HCl}$ doped polianiline:nylon-6,6-(5) composite films 
ite at different magnifications. Evidently, the homogeneous formation of composites of polyaniline in the nylon-6,6 matrix is observed. The surface morphology of chemically prepared PANI showed a cloudy structure while that of nylon- 6,6 is somewhat granular and the morphology of composite film is totally different from their parent compounds i. e. PANI and nylon-6,6. The difference in surface morphology of composite indicates the binding of the parent component consequently, the formation of a composite.

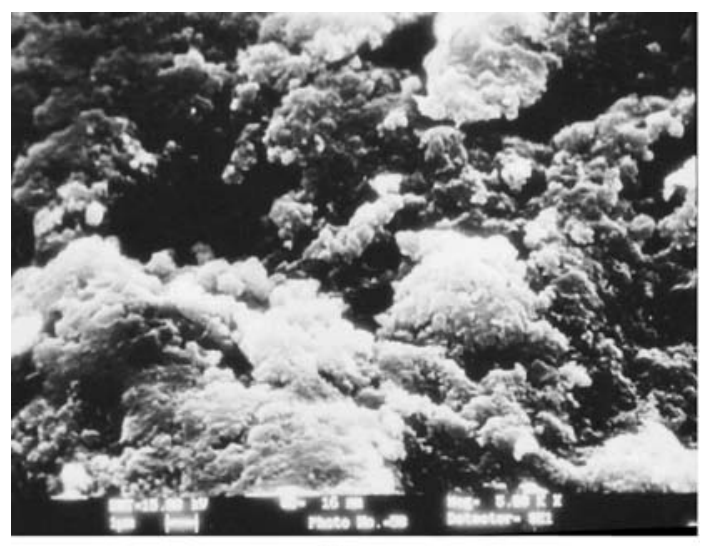

a)

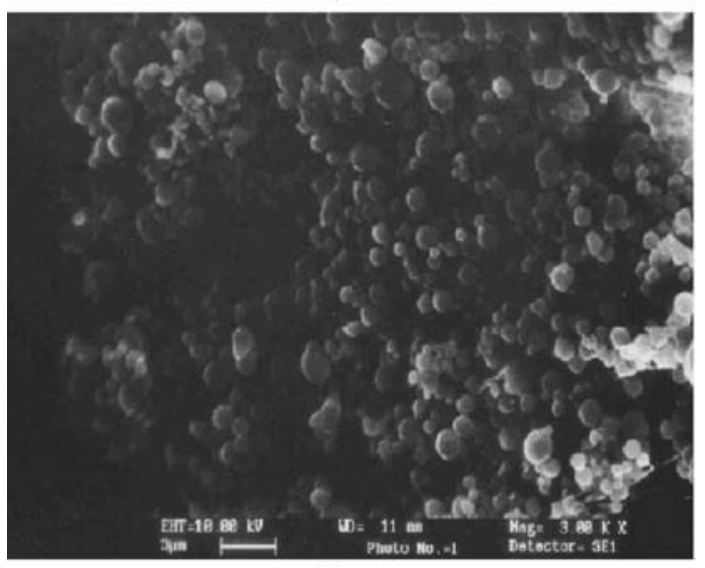

b)

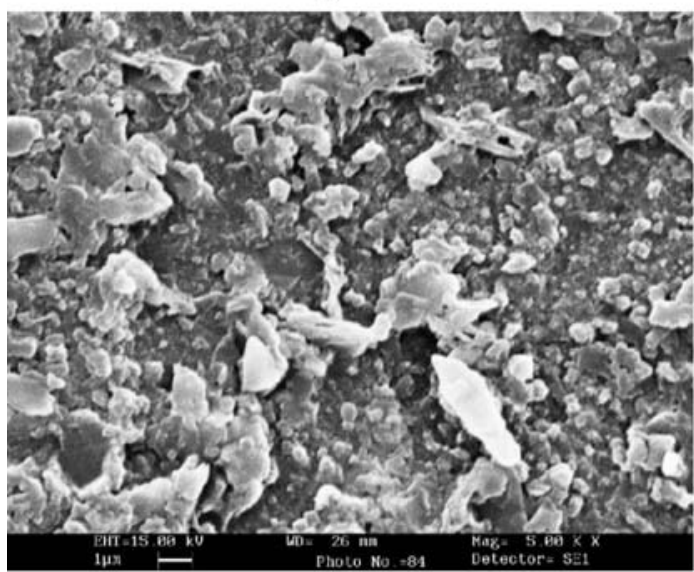

c)

Figure 1. SEM photograph of a) polyaniline, b) nylon-6,6 and c) of PANI:Ny-6,6-(5) composite film

\subsubsection{FTIR studies}

The FTIR spectra of polyaniline:nylon- 6,6 composites are presented in Figure 2. The band corresponding to out of plane bending vibration of $\mathrm{C}-\mathrm{H}$ bond of p-disubstituted benzene rings appears at $824 \mathrm{~cm}^{-1}$. The bands corresponding to stretching vibration of $\mathrm{N}-\mathrm{B}-\mathrm{N}$ and $\mathrm{N}=\mathrm{Q}=\mathrm{N}$ structures appear at $1497 \mathrm{~cm}_{-1}$ and $1587 \mathrm{~cm}^{-1}$ respectively where $-\mathrm{B}-$ and $=\mathrm{Q}=$ stand for benzenoid and quinoid moieties in the polymer. The bands corresponding to vibration mode of $\mathrm{N}=\mathrm{Q}=\mathrm{N}$ ring and stretching mode of $\mathrm{C}-\mathrm{N}$ bond appear at 1143 and $1302 \mathrm{~cm}^{-1}$. The band close to $1130 \mathrm{~cm}^{-1}$ is described as being characteristic of the conducting polymer due to the delocalization of electrical charges caused by deprotonation. The FTIR spectrum supports the presence of benzenoid as well as quinoid moieties in the polyaniline. The characteristic bands at around $688,1642,3303 \mathrm{~cm}^{-1}$ attributed to nylon6,6 are present in all the composites. As-prepared polyaniline: nylon- 6,6 composite showed a strong

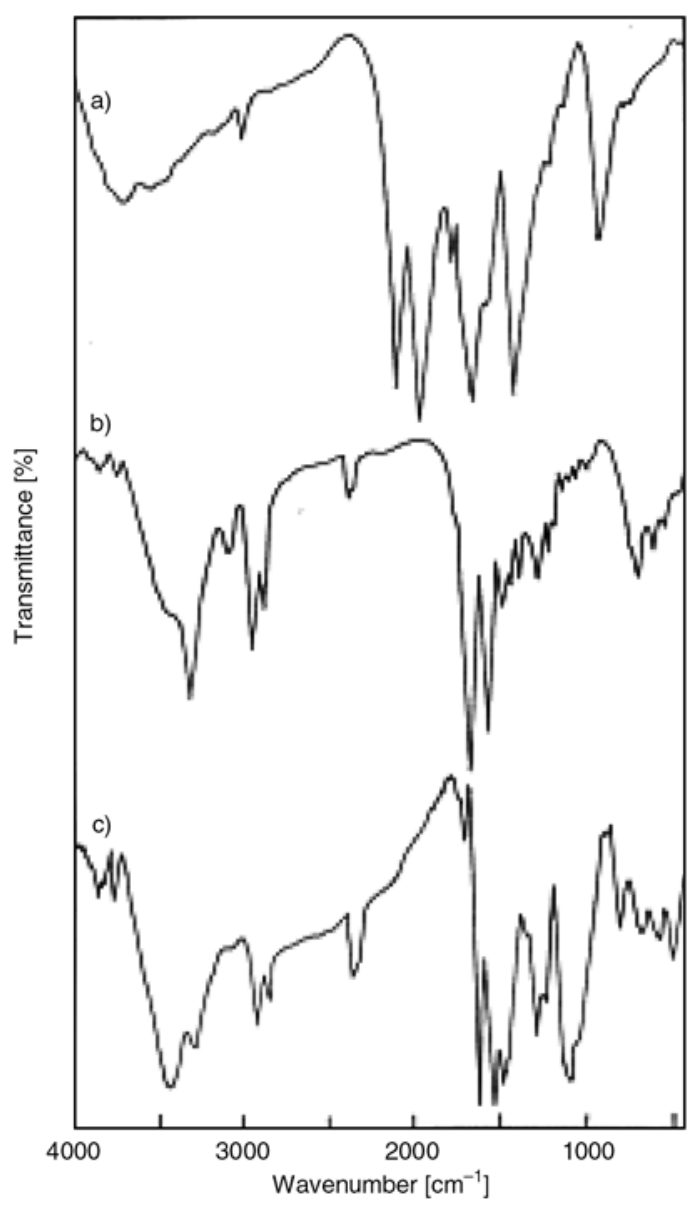

Figure 2. FTIR spectra of polyaniline:nylon-6,6 composite films. a) PANI:Ny-6,6-(3), b) PANI:Ny-6,6-(4) and c) PANI:Ny-6,6-(5) 
band around $1650 \mathrm{~cm}^{-1}$ corresponding to carbonyl group of nylon-6,6. For polyaniline, the band corresponding to out of plane bending vibration of $\mathrm{C}-\mathrm{H}$ bond of p-disubstituted benzene ring appears around $824 \mathrm{~cm}^{-1}$. The bands corresponding to stretching vibration of $\mathrm{N}-\mathrm{B}-\mathrm{N}$ and $\mathrm{N}=\mathrm{Q}=\mathrm{N}$ structure appear around $1377 \mathrm{~cm}^{-1}$ and $1500 \mathrm{~cm}^{-1}$ respectively (where $-\mathrm{B}-$ and $=\mathrm{Q}=$ stand for benznoid and quinoid moieties in the polymer). The band corresponding to stretching mode of $\mathrm{C}-\mathrm{N}$ bond appears at $1504 \mathrm{~cm}^{-1}$ [19]. The gradual increase in the intensities of the bands corresponding to polyaniline and decrease in the intensities of bands corresponding to nylon-6,6 support the gradual change in the composition of the composite samples.

\subsubsection{Stability in terms of DC electrical conductivity retention}

Three samples, PANI:Ny-6,6-(3), PANI:Ny-6,6-(4) and PANI:Ny-6,6-(5) were selected for the study of thermal stability in terms of DC electrical conductivity retention. It was observed that the electrical conductivity of PANI:Ny-6,6 composites increased on exposure to $\mathrm{HCl}$, due to doping of polyaniline component of the composite films, as evident from Equation (10) [20, 21]:

$$
\begin{aligned}
& \text { PANI }(\mathrm{Ny}-6,6)+n \mathrm{HCl} \\
& \quad \longrightarrow\left[\left(\mathrm{PANI}-n \mathrm{H}^{+}(\mathrm{Ny}-6,6)\right]\left[\left(n \mathrm{Cl}^{-}\right)\right]\right.
\end{aligned}
$$

The isothermal stability testing of the $\mathrm{HCl}$ doped films in terms of DC electrical conductivity retention was carried out at $50,70,90,110$ and $130^{\circ} \mathrm{C}$ in an air oven. The electrical conductivity measurements were done at an interval of 10 minutes in the accelerated ageing experiments. The electrical conductivity measured with respect to time of accelerated ageing is presented in Figure 3. The thermal stability was also studied by repeatedly measuring DC electrical conductivity with increasing temperature from 30 to $130^{\circ} \mathrm{C}$ for five times at an interval of 40 minutes.

The electrical conductivity of the polyaniline: nylon-6,6 films was measured from 30 to $130^{\circ} \mathrm{C}$ and found to be in the semi-conducting region. All the composite films followed the Arhenius equation for the temperature dependence of the electrical conductivity from 30 to $115^{\circ} \mathrm{C}$. After that a deviation in electrical conductivity was observed.
A combination of all or some of the following factors could be responsible for the decrease in electrical conductivity beyond $130^{\circ} \mathrm{C}$ such as the loss of dopant and degradation, the chemical reaction of dopant with polyaniline or nylon- 6,6 , the semi-conductor to metal transition and the approaching of $T_{g}$. The studies on the stability of electrical conductivity under isothermal conditions at 50, 70, 90, 110 and $130^{\circ} \mathrm{C}$ showed that the electrical conductivity is stable at 50,70 and $90^{\circ} \mathrm{C}$ supporting the fact that all the composite films were sufficiently stable in term of DC electrical conductivity retention under ambient conditions below $90^{\circ} \mathrm{C}$ and the electrical conductivity decreases with respect to time at 110 and $130^{\circ} \mathrm{C}$ may be attributed to the loss of dopant. The stability in terms of DC electrical conductivity retention was observed to be fairly good as studied

a)
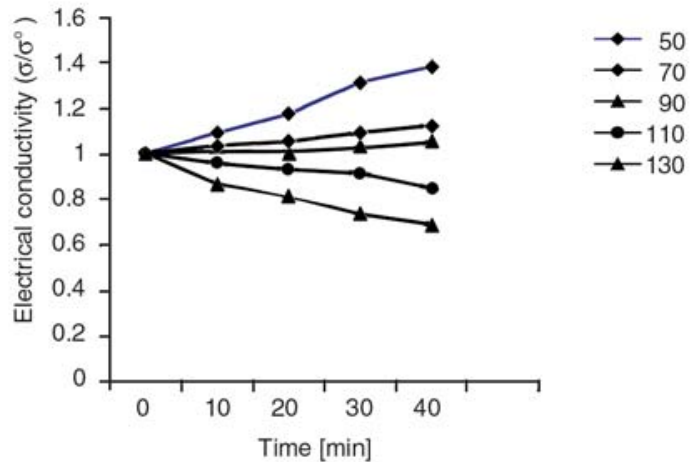

b)
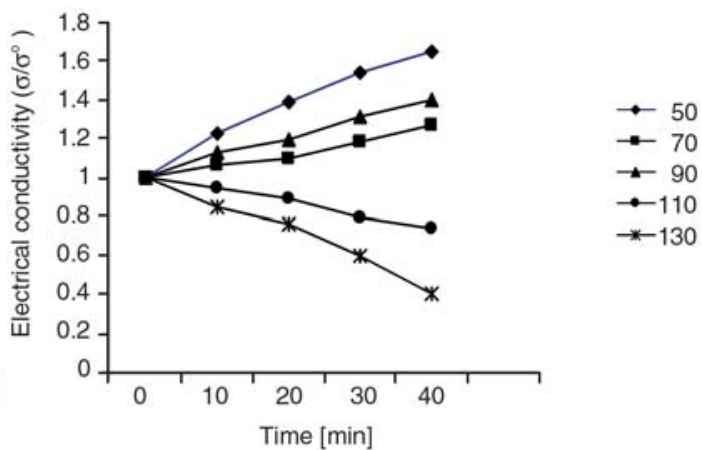

$₫ 90$

$\rightarrow-110$

$\rightarrow-130$

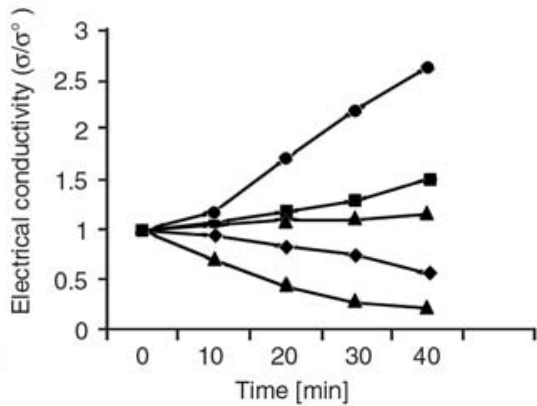

$\rightarrow 50$

$\rightarrow \quad 70$

$\rightarrow 90$

$\longrightarrow-110$
$\longrightarrow-130$

Figure 3. Stability in terms of DC electrical conductivity retention under isothermal conditions at 50, 70, 90, 110 and $130^{\circ} \mathrm{C}$. a) PANI:Nylon-6,6-(3), b) PANI:Nylon-6,6-(4) and c) PANI:Nylon-6,6-(5) 
by isothermal technique. The polyaniline:nylon-6,6 composite films were found to be suitable for use in electrical and electronic applications below $90^{\circ} \mathrm{C}$ under ambient conditions.

\section{Conclusions}

The preparation of conductive polyaniline:nylon6,6 composite films is very successfully demonstrated by diffusion of aniline monomer in to the nylon-6,6 matrix followed by oxidative polymerization of aniline within the nylon- 6,6 matrix technique. Thus prepared polyaniline:nylon- $6,6 \mathrm{com}-$ posite films possess high electrical conductivities at higher doping levels. It is also observed that the electrical conductivity of polyaniline:nylon-6,6 films increases with increase in polyaniline content of the composite. The composite material was successfully characterized for their electrical properties, FTIR and SEM. Thermal stability in term of DC electrical conductivity retention is fairly good as studied by several experimental techniques. Most of the formulations of the composites so prepared are suitable for use in electrical and electronic applications below $90^{\circ} \mathrm{C}$ under ambient conditions.

\section{Acknowledgements}

One of the authors (MK) is thankful to Mr Inamuddin for his suggestions.

\section{References}

[1] Das N. C., Shinchi Y., Masamichi H., Chaki T. K.; Khastgir D., Chakraborty A.: Electrical conductivity and electromagnetic interference shielding effectiveness of polyaniline-ethylene vinyl acetate composite. Polymer International, 54, 256-259 (2005).

[2] Planés J., Wolter A., Cheguetting Y., Pron A., Genound F., Nectschein M.: Transport properties of polyaniline cellulose acetate blends. Physical Review B, 58, 7774-7785 (1998).

[3] Ragunathan A., Kahol P. K., Ho J. C., Chen Y. Y., Dao Y. D., Lin Y. S.: Low temperature heat capacities of polyaniline and polyaniline methylemathacrylate blends. Physical Review B, 58, 15955-15958 (1998).

[4] Sanjai B., Ragunathan A., Natrajan T. S., Rangarajan G., Thomas S., Prabhakaran P. V., Venkatachalam S.: Charge transport and magnetic properties doped with methanesulfonic acid. Physics Review B, 55, 10734 10744 (1997).
[5] Han M. G., Bun S. W., Im S. S.: Thermal stability study of conductive polyaniline/polyimide blend film on their conductivity and ESR measurements. Polymers for Advanced Technology, 13, 320-328 (2002).

[6] Ahmed A. A., Inamuddin: Electrically conducting polymer composites. in 'Specialty Polymers: Materials and Applications' (Ed.: F. Mohammad) I. K. International, New Delhi, 535-583 (2007).

[7] Tchmutin I. A., Ponomarenko A. T., Krinichnaya E. P., Kozub G. I., Efimov O. N.: Electrical properties of composite based on conjugated polymer and conductive fillers. Carbon, 41, 1391-1395 (2003).

[8] Srinivasan D., Natrajan T. S., Rangrajan G., Bhat S. B., Weslling B.: Electron spin resonance absorption in organic metal polyaniline and its blend with PMMA. Solid State Communications, 110, 503-508 (1999).

[9] Ahmed A. A., Mohammad F.: Studies on polyaniline:polyethyleneterphthalate films. Solid State Phenomena, 111, 99-102 (2006).

[10] Yam W. Y., Ismail J., Kammer H. W., Lechner M. D., Kummerlowe C.: Thermal properties of poly (styreneblock- $\varepsilon$-caprolactone) in blends with poly (vinyle methyle ether). Polymer, 41, 9073-9080 (2000).

[11] Hsu C-H., Vaca-Segonds P., Epstein A. J.: Polyaniline/PPD-T fibres. Synthetic Metals, 41, 1005-1008 (1991).

[12] Kang E. T., Neoh K. G., Tan K. L.: Polyaniline: a polymer with many interesting intrinsic redox states. Progress in Polymer Science, 23, 277-324 (1998).

[13] Ahmed A. A., Mohammad F.: Studies on electrically conducting polyaniline: nylon- 6,6 composites: a potential material for electrical and electronic applications. Journal of Solid State Phenomena, 111, 95-98 (2006).

[14] MacDiarmid A. G., Epstein A.: Secondary doping in polyaniline. Synthetic Metals, 69, 85-92 (1995).

[15] Schöllhorn R., Zagefka H-D.: Demonstration of the ionic structure of the intercalation compound $\mathrm{TaS}_{2}$ $\mathrm{NH}_{3}$. Angewandte Chemie International Edition in English, 16, 199-200 (1997).

[16] Foot P. J. S., Shaker N. G.: Amine intercalates of lamellar compounds $\mathrm{NiPS}_{3}$ and $\mathrm{CdPS}_{3}$. Materials Research Bulletin, 18, 173-180 (1983).

[17] Mohammad F.: Compensation behavior of electrically conductive polythiophene and polypyrrole. Journal of Physics D: Applied Physics, 31, 951-959 (1998).

[18] Sung W. B., Soon S. I.: Physical properties and doping charecterstic of polyaniline-nylon 6 composite films. Polymer, 39, 485-489 (1998).

[19] Rao C. N. R.: Chemical application of infrared spectroscopy. Academic Press, New York (1963).

[20] MacDiarmid A. G.: Synthetic metals: a novel role for organic polymers. Synthetic Metals, 125, 11-22 (2002).

[21] Heeger A. J.: Semicoducting and metallic polymers: the forth generation of polymeric materials. Synthetic Metals, 125, 23-42 (2002). 